QUARTERLY OF APPLIED MATHEMATICS

VOLUME LXVII, NUMBER 2

JUNE 2009, PAGES 201-220

S 0033-569X(09)01092-8

Article electronically published on February 18, 2009

\title{
STABILITY OF RANDOM-SWITCHING SYSTEMS OF DIFFERENTIAL EQUATIONS
}

\author{
BY \\ C. ZHU (Department of Mathematical Sciences, University of Wisconsin-Milwaukee, Milwaukee, \\ Wisconsin 53201), \\ G. YIN (Department of Mathematics, Wayne State University, Detroit, Michigan 48202), \\ AND \\ Q. S. SONG (Department of Mathematics, University of Southern California, Los Angeles, \\ California 90089)
}

\begin{abstract}
This work is devoted to the stability of random-switching systems of differential equations. After presenting the formulation of random-switching systems, the notion of stability is recalled, and sufficient conditions in terms of the Liapunov function are presented. Then easily verifiable conditions for stability and instability of systems arising in approximation are established. Using a logarithm transformation, necessary and sufficient conditions are derived for systems that are linear in the continuous state component. Several examples are provided as demonstrations. Among other things, a somewhat different behavior from the well-known Hartman-Grobman theorem is observed.
\end{abstract}

1. Introduction. Inspired by the original work [4 on piecewise deterministic dynamic systems that are formulated as differential equations modulated by a randomswitching (also known as regime-switching) process, growing attention has been drawn to such dynamic systems. This is because of the increasing demand for modeling largescale and complex systems, designing optimal controls, and carrying out optimization

Received September 6, 2007.

2000 Mathematics Subject Classification. Primary 60J27, 60J75, 93D05, 93D20, 93E15.

Key words and phrases. Random switching, hybrid system, stability, necessary condition, sufficient condition.

Research of the first author was supported in part by the National Science Foundation under DMS0304928.

Research of the second author was supported in part by the National Science Foundation under DMS0603287, and in part by the National Security Agency under MSPF-068-029.

Research of the third author was supported in part by the U.S. Army Research Office MURI grant W911NF-06-1-0094 at the University of Southern California.

E-mail address: zhu@uwm.edu

E-mail address: gyin@math.wayne.edu

E-mail address: qingshus@usc.edu

(C)2009 Brown University Reverts to public domain 28 years from publication 
tasks. A distinctive feature of these systems is the coexistence of continuous dynamics and discrete events, so they are often called stochastic hybrid systems (or for short hybrid systems). In this paper, we consider hybrid systems modulated by a random-switching process, which are "equivalent" to a number of ordinary differential equations coupled by a switching or jump component.

With the presence of both continuous dynamics and discrete events, hybrid systems are capable of describing complex systems and their inherent uncertainty and randomness in the environment. The hybrid formulation provides more opportunity for realistic models, but adds much more difficulty in analyzing the underlying systems. One class of such systems is the hybrid systems with Markovian switching, also known as the randomswitching systems. Such systems have been found in emerging applications of systems and controls, financial engineering, wireless communications, manufacturing systems, and other related fields; see for example, [1, 12, 16, 19] and the many references therein. Random-switching processes are used to model the demand rate or machine capacity in production planning, they are used to describe the volatility changes over time to capture discrete shifts such as market trends and interest rates, etc. (for example, a two-state Markov chain can be used to characterize the up and down trends of a market), and they are used to model the time-varying parameter over time for network problems.

Many real-world systems are in operation for a long period of time. As a result, the longtime behavior of such systems is of foremost importance. Recently, much attention has been drawn to the study of the stability of such systems; see 2, 17, 11, 20] among others. Much of the contemporary study of stochastic stability of dynamic systems can be traced back to the original work [6], in which a systematic approach was developed for the stability of systems with Markovian switching using Liapunov function methods. This important work stimulated much of the subsequent developments.

Due to the increasing need of random environment models, the stability of hybrid systems has received resurgent attention lately. An effort has been placed on deriving more easily verifiable conditions for stability and instability. This work also aims to contribute in this direction. One of the main features of $[\underline{6}$ is to use quadratic Liapunov functions to obtain verifiable conditions for stability of the system $\dot{x}(t)=A(\gamma(t)) x(t)$, where $\gamma(t)$ is a continuous-time Markov chain with a constant generator $Q$. Their conditions amount to solving a system of linear nonhomogeneous equations, which is generally complicated. In this work, we present new easily verifiable conditions for stability and instability with the aid of nonquadratic Liapunov functions. Compared with the conditions in $\underline{6}$, our conditions in Theorem 3.2. Corollary 3.3, and Corollary 3.4 are simpler and easier to verify. Moreover, our results can be applied to more general models; see Section 2 for more details.

For general stochastic stability theory of stochastic differential equations without switching, we refer the reader to the profound work by Khasminskii [8], among others. In lieu of considering diffusion processes as in [8], this paper is devoted to the stability of random-switching systems of differential equations.

For random-switching systems, we first obtain sufficient conditions for stability and instability. Our approach leads to a necessary and sufficient condition for systems whose 
continuous component is one dimensional. For multi-dimensional systems, our conditions involve the use of minimal and maximal eigenvalues of appropriate matrices. The difference between the maximal and minimal eigenvalues results in a gap for the stability conditions of multi-dimensional systems. To close the gap, we introduce a logarithm transformation leading to the continuous component taking values in unit spheres. This, in turn, enables us to obtain necessary and sufficient conditions for stability.

Since the systems we are interested in have continuous components (representing continuous dynamics) as well as discrete components (representing the discrete events), their asymptotic behavior can be quite different from a single system of differential equations. As noted, a random-switching differential system may be considered as several differential equations connected through the switching process. We show that even though some of the individual equations are not stable, the entire switching system may still be stable as long as certain conditions are satisfied.

For random switching systems that are linear in their continuous component, suppose that some of the associated differential equations are stable and the others are not. If the jump component is ergodic, we show that as long as the stable part of the differential equations dominates the rest (in an appropriate sense, which will be made precise), the coupled hybrid system will be stable. For nonlinear differential equations, the wellknown Hartman-Grobman theorem (see [13, Section 2.8]) provides an important result concerning the local qualitative behavior. It says that near a hyperbolic equilibrium point $x_{0}$, the nonlinear system $\dot{x}=f(x)$ has the same qualitative structure as that of the linear system $\dot{x}=\nabla f\left(x_{0}\right) x$, while the topological equivalence may not hold for a nonhyperbolic equilibrium point (e.g., a center). In treating hybrid systems, consider the differential equations $\dot{x}=f(x, \gamma(t))$ and $\dot{x}=\nabla f\left(x_{0}, \gamma(t)\right) x$ for $\gamma(t)$ being in a finite set. We shall show that although some of the linear equations have centers, as long as the spectrum of the coefficients of the differential equation corresponding to the stable node dominates that of the centers, the overall system will still be topologically equivalent to the linear (in a continuous component) system. To reveal the salient features, we present a number of examples and display the corresponding phase portraits. The results are quite revealing.

The rest of the paper is arranged as follows. Section 2 begins with the formulation of the random-switching systems and provides definitions of stability, instability, and asymptotical stability of the equilibrium point of the random-switching hybrid systems and gives some preliminary results. For the purpose of our asymptotic analysis, we also present sufficient conditions for stability, instability, and asymptotical stability. Easily verifiable conditions for stability and instability of the systems are provided in Section 3. Section 4 presents a sharper condition for systems that are linear in the continuous state variable. To demonstrate our results, we provide several examples in Section 5. Finally, we conclude the paper with further remarks in Section 6 .

\section{Formulation and preliminary results.}

2.1. Problem setup. Throughout the paper, we use $z^{\prime}$ to denote the transpose of $z \in$ $\mathbb{R}^{\ell_{1} \times \ell_{2}}$ with $\ell_{i} \geq 1$, whereas $\mathbb{R}^{\ell \times 1}$ is simply written as $\mathbb{R}^{\ell} ; \mathbb{1}=(1,1, \ldots, 1)^{\prime} \in \mathbb{R}^{m}$ is a column vector with all entries being 1 ; the Euclidean norm for a row or column vector $x$ 
is denoted by $|x|$. As usual, $I$ denotes the identity matrix. For a matrix $A$, its trace norm is denoted by $|A|=\sqrt{\operatorname{tr}\left(A^{\prime} A\right)}$. When $B$ is a set, $I_{B}(\cdot)$ denotes the indicator function of $B$. For $A \in \mathbb{R}^{n \times n}$ being a symmetric matrix, we use $\lambda_{\max }(A)$ and $\lambda_{\min }(A)$ to denote the maximum and minimum eigenvalue of $A$, respectively.

Consider the system with random switching

$$
\dot{x}(t)=f(x(t), \gamma(t)), \quad x(0)=x \in \mathbb{R}^{n}, \quad \gamma(0)=\gamma \in \mathcal{M},
$$

where $x(t)$ is the continuous state, $f(\cdot, \cdot): \mathbb{R}^{n} \times \mathcal{M} \mapsto \mathbb{R}^{n}$, and $\gamma(\cdot)$ is a jump process taking value in a finite state space $\mathcal{M}=\{1,2, \ldots, m\}$ with a generator $Q(x)=\left(q_{i j}(x)\right)$ satisfying $q_{i j}(x) \geq 0$ for $j \neq i$ and $\sum_{j \in \mathcal{M}} q_{i j}(x)=0$ for all $x \in \mathbb{R}^{n}$ and $i \in \mathcal{M}$. The evolution of the jump component is described by

$$
\mathbf{P}\{\gamma(t+\Delta t)=j \mid \gamma(t)=i,(x(s), \gamma(s)), s \leq t\}=q_{i j}(x(t)) \Delta t+o(\Delta t), \quad i \neq j .
$$

Note that in our formulation, the $x$-dependent $Q(x)$ is considered, where in [2, 6, 11, 20, the constant generator $Q$ was used.

The consideration of switching ordinary differential equations of the form (2.1) comes from a wide variety of applications including control, optimization, estimation, and tracking. For example, in [16, with the motivation of using stochastic recursive algorithms for tracking Markovian parameters such as those in spreading code optimization in CDMA (Code Division Multiple Access) wireless communication, we used an adaptive algorithm with constant step size to construct a sequence of estimates of the time-varying distribution. It is shown for the first time that under simple conditions, a continuous-time interpolation of the iteration converges weakly, not to an ODE as is widely known in the literature of stochastic approximation [10, but to a system of ODEs with regime switching. Subsequently, by treating least squares type algorithms involving Markovian jump processes in [15, random switching ODEs were also obtained. Thus, not only is the study of systems given by (2.1) and (2.2) of mathematical interest, but it also provides practical guidance for many applications.

Associated with the process $(x(t), \gamma(t))$ defined by (2.1)-(2.2), there is an operator $\mathcal{L}$ defined as follows. For each $i \in \mathcal{M}$ and any $g(\cdot, i) \in C^{1}\left(\mathbb{R}^{n}\right)$,

$$
\mathcal{L} g(x, i)=\langle f(x, i), \nabla g(x, i)\rangle+Q(x) g(x, \cdot)(i),
$$

where $\langle\cdot, \cdot\rangle$ is the usual inner product in $\mathbb{R}^{n}, \nabla g(x, i)$ denotes the gradient (with respect to the variable $x)$ of $g(x, i)$, and

$$
Q(x) g(x, \cdot)(i)=\sum_{j \in \mathcal{M}} q_{i j}(x) g(x, j) .
$$

For further references on the associated operator (or generator) of the hybrid system (2.1) -(2.2), we refer the reader to [6]; see also the related work [14.

To proceed, we need conditions regarding the smoothness and growth of the functions involved, and the condition that 0 is an equilibrium point of the dynamic system. Hence we assume that the following hypotheses are valid throughout the paper:

(A1) The matrix-valued function $Q(\cdot)$ is bounded and continuous.

(A2) The functions $f(\cdot, \gamma)$ are locally Lipschitz continuous and satisfy $f(0, \gamma)=0$, for each $\gamma \in \mathcal{M}$. 
(A3) There exists a constant $K_{0}>0$ such that for each $\gamma \in \mathcal{M}$,

$$
|f(x, \gamma)| \leq K_{0}(1+|x|), \quad \text { for all } x \in \mathbb{R}^{n} .
$$

It is well known that under these conditions, system (2.1)-(2.2) has a unique solution; see 14 for details. In what follows, a process starting from $(x, \gamma)$ will be denoted by $y^{x, \gamma}(t)=\left(x^{x, \gamma}(t), \gamma^{x, \gamma}(t)\right)$ to emphasize the dependence on the initial condition. If the context is clear, we simply write $y(t)=(x(t), \gamma(t))$.

2.2. Preliminary results. In this subsection, we first recall the definitions of stability, instability, asymptotic stability, and exponential $p$-stability. Then we present some preparatory results of stability and instability in terms of Liapunov functions.

Definition 2.1 ([6]). The equilibrium point $x=0$ of system (2.1)-(2.2) is said to be

(i) stable in probability, if for any $r>0$,

$$
\lim _{x \rightarrow 0} \mathbf{P}\left\{\sup _{t \geq 0}\left|x^{x, \gamma}(t)\right|>r\right\}=0,
$$

and it is said to be unstable in probability if it is not stable in probability.

(ii) asymptotically stable in probability, if it is stable in probability and

$$
\lim _{x \rightarrow 0} \mathbf{P}\left\{\lim _{t \rightarrow \infty} x^{x, \gamma}(t)=0\right\}=1 .
$$

(iii) exponentially p-stable, if for some positive constants $K$ and $\sigma$,

$$
\mathbf{E}\left|x^{x, \gamma}(t)\right|^{p} \leq K|x|^{p} \exp \{-\sigma t\}, \quad \text { for any } \gamma \in \mathcal{M} .
$$

To study the stability of the equilibrium point $x=0$, we first observe that almost all trajectories of the system (2.1) -(2.2) starting from a nonzero state will never reach the origin.

Proposition 2.2. Let conditions (A1)-(A3) be satisfied. Then

$$
\mathbf{P}\left\{x^{x, \gamma}(t) \neq 0, t \geq 0\right\}=1, \quad \text { for any }(x, \gamma) \in \mathbb{R}^{n} \times \mathcal{M} \text { with } x \neq 0 .
$$

Proof. The assertion of the proposition can be proved using a slight modification of the argument in [11, Lemma 2.1]. The details are omitted.

In view of (2.6), we can work with functions $V(\cdot, i), i \in \mathcal{M}$, which are continuously differentiable in the deleted neighborhood of 0 in what follows. This turns out to be quite convenient. Another immediate and interesting consequence of (2.6) is the following $L^{p}$ estimate for the solution of the system (2.1)-(2.2). The result is interesting in its own right.

Theorem 2.3. Let conditions (A1)-(A3) be satisfied. Then for any $p \geq 1$ and any $(x, \gamma) \in \mathbb{R}^{n} \times \mathcal{M}$ with $x \neq 0$, we have

$$
\mathbf{E}\left|x^{x, \gamma}(t)\right| \leq\left(|x|^{p}+\frac{1}{2}\right) \exp \left(2 p m K_{0} t\right)-\frac{1}{2} \leq\left(|x|^{p}+\frac{1}{2}\right) \exp \left(2 p m K_{0} t\right) .
$$

Proof. For each $\gamma \in \mathcal{M}$, the function $V(x, \gamma)=|x|^{p}$ is continuously differentiable in the domain $|x|>\delta$ for any $\delta>0$. Let $\tau_{\delta}$ be the first exit time of the process $x^{x, \gamma}(\cdot)$ from 
the set $\left\{x \in \mathbb{R}^{n}:|x|>\delta\right\} \times \mathcal{M}$. That is,

$$
\tau_{\delta}:=\inf \left\{t \geq 0:\left|x^{x, \gamma}(t)\right| \leq \delta\right\} .
$$

For any $t>0$, set $\tau_{\delta}(t):=\min \left\{\tau_{\delta}, t\right\}$. Then it follows from the Cauchy-Schwartz inequality and (2.5) that

$$
\begin{aligned}
\mathbf{E}\left|x^{x, \gamma}\left(\tau_{\delta}(t)\right)\right|^{p}= & |x|^{p}+\mathbf{E} \int_{0}^{\tau_{\delta}(t)} p\left|x^{x, \gamma}(s)\right|^{p-2}\left\langle x^{x, \gamma}(s), f\left(x^{x, \gamma}(s), \gamma^{x, \gamma}(s)\right)\right\rangle d s \\
\leq & |x|^{p}+p m K_{0} \mathbf{E} \int_{0}^{\tau_{\delta}(t)}\left|x^{x, \gamma}(s)\right|^{p-1}\left(1+\left|x^{x, \gamma}(s)\right|\right) d s \\
\leq & |x|^{p}+2 p m K_{0} \mathbf{E} \int_{0}^{\tau_{\delta}(t)}\left|x^{x, \gamma}(s)\right|^{p} d s \\
& \quad+p m K_{0} \mathbf{E} \int_{0}^{\tau_{\delta}(t)}\left|x^{x, \gamma}(s)\right|^{p-1} I_{\left\{\delta \leq\left|x^{x, \gamma}(s)\right|<1\right\}} d s \\
\leq & |x|^{p}+2 p m K_{0} \mathbf{E} \int_{0}^{\tau_{\delta}(t)}\left(\left|x^{x, \gamma}(s)\right|^{p}+\frac{1}{2}\right) d s .
\end{aligned}
$$

Note that for all $s \leq \tau_{\delta}(t)$, we have $s=\tau_{\delta}(s)$. Hence we have

$$
\begin{aligned}
\mathbf{E}\left|x^{x, \gamma}\left(\tau_{\delta}(t)\right)\right|^{p} & \leq|x|^{p}+2 p m K_{0} \mathbf{E} \int_{0}^{\tau_{\delta}(t)}\left(\left|x^{x, \gamma}\left(\tau_{\delta}(s)\right)\right|^{p}+\frac{1}{2}\right) d s \\
& \leq|x|^{p}+2 p m K_{0} \int_{0}^{t}\left(\mathbf{E}\left|x^{x, \gamma}\left(\tau_{\delta}(s)\right)\right|^{p}+\frac{1}{2}\right) d s .
\end{aligned}
$$

Applying Gronwall's inequality to $\left[\mathbf{E}\left|x^{x, \gamma}\left(\tau_{\delta}(t)\right)\right|^{p}+(1 / 2)\right]$ implies that

$$
\mathbf{E}\left|x^{x, \gamma}\left(\tau_{\delta}(t)\right)\right|^{p}+\frac{1}{2} \leq\left(|x|^{p}+\frac{1}{2}\right) \exp \left(2 p m K_{0} t\right),
$$

or equivalently,

$$
\begin{aligned}
\mathbf{E}\left|x^{x, \gamma}\left(\tau_{\delta}(t)\right)\right|^{p} & \leq\left(|x|^{p}+\frac{1}{2}\right) \exp \left(2 p m K_{0} t\right)-\frac{1}{2} \\
& \leq\left(|x|^{p}+\frac{1}{2}\right) \exp \left(2 p m K_{0} t\right) .
\end{aligned}
$$

Note that we have from (2.6) that

$$
\tau_{\delta}(t) \rightarrow t \text { as } \delta \rightarrow 0 \text { with probability } 1 \text { for any } t>0 .
$$

Finally, letting $\delta \rightarrow 0$ in (2.9), by virtue of Fatou's Lemma, we obtain (2.7).

REMARK 2.4. If condition (2.5) is replaced by

$$
|f(x, \gamma)| \leq K|x|, \quad \text { for all }(x, \gamma) \in \mathbb{R}^{n} \times \mathcal{M},
$$

where $K$ is some positive constant, then the conclusion of Theorem 2.3 can be strengthened to the following. For any $\beta \in \mathbb{R}$ and any $(x, \gamma) \in \mathbb{R}^{n} \times \mathcal{M}$ with $x \neq 0$, we have

$$
\mathbf{E}\left|x^{x, \gamma}(t)\right|^{\beta} \leq|x|^{\beta} e^{\rho t},
$$

where $\rho$ is a constant depending only on $\beta, m$, and the constant $K$ given in (2.10). 
In fact, by virtue of (2.10), we obtain by a slight modification of the argument in the proof of Theorem 2.3 that

$$
\mathbf{E}\left|x^{x, \gamma}\left(\tau_{\delta}(t)\right)\right|^{\beta} \leq|x|^{\beta} e^{\rho t}
$$

where $\rho$ is a constant depending only on $\beta, m$ and the constant $K$ in (2.10). Then similar to the proof of Theorem 2.3. (2.11) follows from (2.6) and Fatou's Lemma.

Next, concerning the stability and asymptotical stability of the equilibrium point $x=0$ of the system (2.1)-(2.2), we have the following results:

Proposition 2.5. Let $D \subset \mathbb{R}^{n}$ be a neighborhood of 0 . Suppose that for each $i \in \mathcal{M}$, there exists a nonnegative function $V(\cdot, i): D \mapsto \mathbb{R}$ such that

(i) $V(\cdot, i)$ is continuous in $D$ and vanishes only at $x=0$;

(ii) $V(\cdot, i)$ is continuously differentiable in $D-\{0\}$ and satisfies

$$
\mathcal{L} V(x, i) \leq 0, \quad \text { for all } x \in D-\{0\} .
$$

Then the equilibrium point $x=0$ is stable in probability.

Proposition 2.6. Assume the conditions of Proposition 2.5. Suppose moreover that for each $i \in \mathcal{M}$, the function $V(\cdot, i)$ satisfies

$$
\begin{aligned}
& \lim _{|x| \rightarrow \infty} V(x, i)=\infty, \\
& \mathcal{L} V(x, i)<0, \text { for all } x \in D-\{0\} .
\end{aligned}
$$

Then the equilibrium point $x=0$ is asymptotically stable in probability.

By virtue of Proposition 2.2, we can slightly modify the arguments in 8 , Theorems 5.3.1 and 5.4.1] to establish Proposition 2.5 and Proposition 2.6. We omit the details here for brevity.

Proposition 2.7. Let $D \subset \mathbb{R}^{n}$ be a neighborhood of 0 . Assume that for each $i \in \mathcal{M}$, there exists a nonnegative function $V(\cdot, i): D \mapsto \mathbb{R}$ such that $V(\cdot, i)$ is continuously differentiable in every deleted neighborhood of 0 , and

$$
\begin{aligned}
& \mathcal{L} V(x, i)<0 \text { for all } x \in D-\{0\}, \\
& \lim _{|x| \rightarrow 0} V(x, i)=\infty, \text { for each } i \in \mathcal{M} .
\end{aligned}
$$

Then the equilibrium point $x=0$ is unstable in probability.

Proof. This proposition follows from a similar argument as that for [8, Theorem 5.4.2]. The detailed proof is omitted.

3. Stability and instability: Sufficient conditions. In the previous section, we obtained sufficient conditions for stability, asymptotical stability, and instability using a Liapunov function argument. Since the results are based on the existence of Liapunov functions, to apply them it is necessary to find appropriate Liapunov functions. Nevertheless, finding suitable Liapunov functions is more often than not a very challenging task. In many applications, it is often more convenient to be able to analyze the stability 
through conditions on the coefficients of the corresponding stochastic differential equations. Thus in this section we continue our study by providing easily verifiable conditions on the coefficients of the system (2.1)-(2.2).

To proceed, we first present the following auxiliary result concerning the solution of a deterministic system of equations. The result, summarized in Lemma 3.1, is crucial for our study in the subsequent sections.

Lemma $3.1(9)$. Let a constant matrix $Q \in \mathbb{R}^{m \times m}$ be the generator of a continuous-time Markov chain $r(t)$ and suppose that $Q$ is irreducible. Denote by $\nu=\left(\nu_{1}, \nu_{2}, \ldots, \nu_{m}\right) \in$ $\mathbb{R}^{1 \times m}$ the unique stationary distribution of $r(t)$. Then the equation

$$
Q c=\eta
$$

has a solution if and only if $\nu \eta=0$, where $c, \eta \in \mathbb{R}^{m}$. Moreover, suppose that $\widetilde{c}_{1}$ and $\widetilde{c}_{2}$ are two solutions of (3.1). Then $\widetilde{c}_{1}-\widetilde{c}_{2}=\alpha_{0} \mathbb{1}$ for some $\alpha_{0} \in \mathbb{R}$.

Note that the irreducibility of $Q$ implies that all the states of the Markov chain belong to the same ergodic class. For multiple ergodic class cases, one may use the idea of twotime-scale formulation and singular perturbation methods as in [17] and [18. To proceed, we assume the following condition holds throughout the rest of the section.

(A4) For each $i \in \mathcal{M}$, there exist $A_{i} \in \mathbb{R}^{n \times n}$ and $\widehat{Q}=\left(\widehat{q}_{i j}\right) \in \mathbb{R}^{m \times m}$, a generator of a continuous-time Markov chain $\widehat{\gamma}(t)$, such that as $x \rightarrow 0$,

$$
\begin{aligned}
& f(x, i)=A_{i} x+o(|x|), \\
& Q(x)=\widehat{Q}+o(1) .
\end{aligned}
$$

Moreover, $\widehat{Q}$ is irreducible. Denote the unique stationary distribution of the associated Markov chain $\widehat{\gamma}(t)$ by $\pi=\left(\pi_{1}, \pi_{2}, \ldots, \pi_{m}\right) \in \mathbb{R}^{1 \times m}$.

Theorem 3.2. Assume conditions (A1)-(A4). Then the following assertions hold:

(i) If there exists a symmetric and positive definite matrix $G$ such that

$$
\sum_{i=1}^{m} \pi_{i} \lambda_{\max }\left(G A_{i} G^{-1}+G^{-1} A_{i}^{\prime} G\right)<0,
$$

then the equilibrium point $x=0$ of the system (2.1) $-(2.2)$ is asymptotically stable in probability.

(ii) If there exists a symmetric and positive definite matrix $G$ such that

$$
\sum_{i=1}^{m} \pi_{i} \lambda_{\min }\left(G A_{i} G^{-1}+G^{-1} A_{i}^{\prime} G\right)>0,
$$

then the equilibrium point $x=0$ of the system (2.1)-(2.2) is unstable in probability.

Proof. (a) We first prove that the equilibrium point $x=0$ of system (2.1)-(2.2) is asymptotically stable in probability if (3.3) holds for some symmetric and positive definite matrix $G$. For notational simplicity, define the column vector $\mu=\left(\mu_{1}, \mu_{2}, \ldots, \mu_{m}\right)^{\prime} \in \mathbb{R}^{m}$ with

$$
\mu_{i}=\frac{1}{2} \lambda_{\max }\left(G A_{i} G^{-1}+G^{-1} A_{i}^{\prime} G\right)
$$


where $G$ is as in (3.3). Also let

$$
\beta:=-\pi \mu=-\sum_{i=1}^{m} \pi_{i} \mu_{i}
$$

Note that $\beta>0$ by (3.3). It follows from assumption (A4) and Lemma 3.1 that the equation

$$
\widehat{Q} c=\mu+\beta \mathbb{1}
$$

has a solution $c=\left(c_{1}, c_{2}, \ldots, c_{m}\right)^{\prime} \in \mathbb{R}^{m}$. Thus we have

$$
\mu_{i}-\sum_{j=1}^{m} \widehat{q}_{i j} c_{j}=-\beta, \quad i \in \mathcal{M}
$$

For each $i \in \mathcal{M}$, consider the Liapunov function

$$
V(x, i)=\left(1-\alpha c_{i}\right)\left(x^{\prime} G^{2} x\right)^{\alpha / 2},
$$

where $0<\alpha<1$ is sufficiently small so that $1-\alpha c_{i}>0$ for each $i \in \mathcal{M}$. It is readily seen that for each $i \in \mathcal{M}, V(\cdot, i)$ is continuous, nonnegative, and vanishes only at $x=0$. In addition, note that since $\alpha>0$ and $1-\alpha c_{i}>0$, we have

$$
\lim _{|x| \rightarrow \infty} V(x, i) \geq \lim _{|x| \rightarrow \infty}\left(1-\alpha c_{i}\right)\left(\lambda_{\min }\left(G^{2}\right)\right)^{\alpha / 2}|x|^{\alpha}=\infty .
$$

Hence, for $i \in \mathcal{M}$, the function $V(\cdot, i)$ satisfies (2.13). A detailed calculation reveals that for $x \neq 0$, we have

$$
\nabla V(x, i)=\left(1-\alpha c_{i}\right) \alpha\left(x^{\prime} G^{2} x\right)^{\alpha / 2-1} G^{2} x .
$$

It yields that for $x \neq 0$,

$$
\begin{aligned}
\mathcal{L} V(x, i) & =\left(1-\alpha c_{i}\right) \alpha\left(x^{\prime} G^{2} x\right)^{\alpha / 2-1} x^{\prime} G^{2}\left(A_{i} x+o(|x|)\right)-\sum_{j \neq i} q_{i j}(x)\left(x^{\prime} G^{2} x\right)^{\alpha / 2} \alpha\left(c_{j}-c_{i}\right) \\
& =\left(1-\alpha c_{i}\right) \alpha\left(x^{\prime} G^{2} x\right)^{\alpha / 2}\left\{\frac{x^{\prime} G^{2} A_{i} x}{x^{\prime} G^{2} x}+o(1)-\sum_{j \neq i} q_{i j}(x) \frac{c_{j}-c_{i}}{1-\alpha c_{i}}\right\} .
\end{aligned}
$$

It follows from condition (A4) that for sufficiently small $|x|$,

$$
\sum_{j \neq i} q_{i j}(x) \frac{c_{j}-c_{i}}{1-\alpha c_{i}}=\sum_{j=1}^{m} q_{i j}(x) c_{j}+\sum_{j \neq i} q_{i j}(x) \frac{c_{i}\left(c_{j}-c_{i}\right)}{1-\alpha c_{i}} \alpha=\sum_{j=1}^{m} \widehat{q}_{i j} c_{j}+O(\alpha)+o(1),
$$

where $o(1) \rightarrow 0$ as $|x| \rightarrow 0$ and $O(\alpha) \rightarrow 0$ as $\alpha \rightarrow 0$. Meanwhile, using the transformation $y=G x$ we have

$$
\begin{aligned}
\frac{x^{\prime} G^{2} A_{i} x}{x^{\prime} G^{2} x} & =\frac{x^{\prime}\left(G^{2} A_{i}+A_{i}^{\prime} G^{2}\right) x}{2 x^{\prime} G^{2} x}=\frac{y^{\prime} G^{-1}\left(G^{2} A_{i}+A_{i}^{\prime} G^{2}\right) G^{-1} y}{2 y^{\prime} y} \\
& \leq \frac{1}{2} \lambda_{\max }\left(G^{-1}\left(G^{2} A_{i}+A_{i}^{\prime} G^{2}\right) G^{-1}\right)=\frac{1}{2} \lambda_{\max }\left(G A_{i} G^{-1}+G^{-1} A_{i}^{\prime} G\right)=\mu_{i} .
\end{aligned}
$$

Moreover, note that

$$
\left(\lambda_{\min }\left(G^{2}\right)\right)^{\alpha / 2}|x|^{\alpha} \leq\left(x^{\prime} G^{2} x\right)^{\alpha / 2} \leq\left(\lambda_{\max }\left(G^{2}\right)\right)^{\alpha / 2}|x|^{\alpha} .
$$


When $|x|<\delta$ with $\delta$ and $0<\alpha<1$ sufficiently small, (3.7)-(3.10) lead to

$$
\mathcal{L} V(x, i) \leq \alpha\left(1-\alpha c_{i}\right)\left(\lambda_{\min }\left(G^{2}\right)\right)^{\alpha / 2}|x|^{\alpha}\left\{\mu_{i}-\sum_{j=1}^{m} \widehat{q}_{i j} c_{j}+o(1)+O(\alpha)\right\} .
$$

Furthermore, by virtue of (3.5), we have

$$
\mathcal{L} V(x, i) \leq \alpha\left(1-\alpha c_{i}\right)\left(\lambda_{\min }\left(G^{2}\right)\right)^{\alpha / 2}|x|^{\alpha}(-\beta+o(1)+O(\alpha)) \leq-\kappa(\varepsilon)<0,
$$

for any $(x, i) \in N_{0} \times \mathcal{M}$ with $|x|>\varepsilon$, where $N_{0} \subset \mathbb{R}^{n}$ is a small neighborhood of 0 and $\kappa(\varepsilon)$ is a positive constant. Therefore we conclude from Proposition 2.6 that the equilibrium point $x=0$ is asymptotically stable in probability.

(b) Now we prove that the equilibrium point $x=0$ is unstable in probability if (3.4) holds for some symmetric and positive definite matrix $G$. Define the column vector $\theta=\left(\theta_{1}, \theta_{2}, \ldots, \theta_{m}\right)^{\prime} \in \mathbb{R}^{m}$ by

$$
\theta_{i}:=\frac{1}{2} \lambda_{\min }\left(G A_{i} G^{-1}+G^{-1} A_{i}^{\prime} G\right),
$$

and set $\delta=-\pi \theta$. Note that

$$
\delta=-\sum_{i=1}^{m} \pi_{i} \theta_{i}<0
$$

As in part (a), assumption (A4), the definition of $\delta$, and Lemma 3.1 imply that the equation $\widehat{Q} c=\theta+\delta \mathbb{1}$ has a solution $c=\left(c_{1}, c_{2}, \ldots, c_{m}\right)^{\prime} \in \mathbb{R}^{m}$ and

$$
\theta_{i}-\sum_{j=1}^{d} \widehat{q}_{i j} c_{j}=-\delta>0, \quad i \in \mathcal{M}
$$

For $i \in \mathcal{M}$, consider the Liapunov function

$$
V(x, i)=\left(1-\alpha c_{i}\right)\left(x^{\prime} G^{2} x\right)^{\alpha / 2},
$$

where $-1<\alpha<0$ is sufficiently small so that $1-\alpha c_{i}>0$ for each $i \in \mathcal{M}$. Similar to the argument in (3.6), we can verify that $V(\cdot, i)$ satisfies (2.16) for each $i \in \mathcal{M}$. Detailed computations as in part (a) show that for any sufficiently small $0<\varepsilon<r$,

$$
\mathcal{L} V(x, i) \leq-\kappa(\varepsilon)<0, \text { for any }(x, i) \in N_{0} \times \mathcal{M} \text { with }|x|>\varepsilon,
$$

where $N_{0} \subset \mathbb{R}^{n}$ is a small neighborhood of 0 and $\kappa(\varepsilon)$ is a positive constant. Therefore Proposition 2.7 implies that the equilibrium point $x=0$ is unstable in probability. This completes the proof of the theorem.

Corollary 3.3. Assume conditions (A1)-(A4). Then the following assertions hold:

(i) The equilibrium point $x=0$ is asymptotically stable in probability if

$$
\sum_{i=1}^{m} \pi_{i} \lambda_{\max }\left(A_{i}+A_{i}^{\prime}\right)<0 .
$$

(ii) The equilibrium point $x=0$ is unstable in probability if

$$
\sum_{i=1}^{m} \pi_{i} \lambda_{\min }\left(A_{i}+A_{i}^{\prime}\right)>0 .
$$


Proof. This corollary follows from Theorem 3.2 immediately by choosing the symmetric and positive definite matrix $G$ in (3.3) and (3.4) to be the identity matrix $I$.

Theorem 3.2 and Corollary 3.3 give sufficient conditions in terms of the maximum and minimum eigenvalues of the matrices for the stability and instability of the equilibrium point $x=0$. Since there is a "gap" between the maximum and minimum eigenvalues, a natural question arises: Can we obtain necessary and sufficient condition for stability? If the component $x(t)$ is 1-dimensional, we have the following result from Theorem 3.2 , which is a necessary and sufficient condition.

Corollary 3.4. Assume conditions (A1)-(A4). Let the continuous component $x(t)$ of the hybrid process $(x(t), \gamma(t))$ given by (2.1)-(2.2) be 1-dimensional. Then the equilibrium point $x=0$ is asymptotically stable in probability if

$$
\sum_{i=1}^{m} \pi_{i} A_{i}<0
$$

and is unstable in probability if

$$
\sum_{i=1}^{m} \pi_{i} A_{i}>0
$$

4. Closing the "gap". This section deals with systems that are linear in the continuous state variable $x$. The system we are interested in is given by

$$
\dot{x}(t)=A(\gamma(t)) x(t),
$$

where $A_{i}=A(i) \in \mathbb{R}^{n \times n}, x(t) \in \mathbb{R}^{n}$, and $\gamma(t)$ is a continuous-time Markov chain with constant generator $Q$. Assume moreover that the Markov chain $\gamma(t)$ is irreducible. Denote the corresponding stationary distribution by $\pi=\left(\pi_{1}, \pi_{2}, \ldots, \pi_{m}\right)$. Our main objective is to obtain a necessary and sufficient condition, or, in other words, to close the gap due to the presence of minimal and maximal eigenvalues as we mentioned in the previous section. Denote the solution of (4.1) by $x(t)$ with initial condition $(x(0), \gamma(0))$. Assume that $x(0) \neq 0$. Then according to Proposition 2.2, $x(t) \neq 0$ for any $t \geq 0$ with probability 1. As a result, $y(t)=x(t) /|x(t)|$ is well defined and takes values on the unit sphere $\mathbb{S}_{n}=\left\{y \in \mathbb{R}^{n}:|y|=1\right\}$. Define $z=\ln |x|$. It is readily seen that

$$
\dot{z}(t)=\frac{\langle x(t), \dot{x}(t)\rangle}{|x(t)|^{2}} .
$$

We shall deal with a case that the Markov chain is fast varying and acting as a 'noise', whereas the $X(t)$ is slowly varying. The noise is averaged out and replaced by its stationary distribution. We suppose that there is a small parameter $\varepsilon>0$ and let $Q=Q^{\varepsilon}=Q_{0} / \varepsilon$, where $Q_{0}$ is a generator and is irreducible. Note that $\alpha(t)$ in this case, should be written as $\alpha^{\varepsilon}(t)$. But for notational simplicity, we suppress the $\varepsilon$ dependence and write it as $\alpha(t)$. 
Using (4.2), for any $T>0$, we have

$$
\begin{aligned}
\frac{z(T)-z(0)}{T} & =\frac{1}{T} \int_{0}^{T} \frac{x^{\prime}(t) A(\gamma(t)) x(t)}{|x(t)|^{2}} d t \\
& =\frac{1}{T} \sum_{i=1}^{m} \int_{0}^{T} \frac{x^{\prime}(t) A_{i} x(t)}{|x(t)|^{2}}\left[I_{\{\gamma(t)=i\}}-\pi_{i}\right] d t+\frac{1}{T} \sum_{i=1}^{m} \int_{0}^{T} \frac{x^{\prime}(t) A_{i} x(t)}{|x(t)|^{2}} \pi_{i} d t .
\end{aligned}
$$

Lemma 4.1. Suppose that $Q_{0}$ is irreducible and that $\varepsilon=o\left(T^{-\Delta}\right)$ as $T \rightarrow \infty$. Then

$$
\frac{1}{T} \sum_{i=1}^{m} \int_{0}^{T} \frac{x^{\prime}(t) A_{i} x(t)}{|x(t)|^{2}}\left[I_{\{\gamma(t)=i\}}-\pi_{i}\right] d t \rightarrow 0 \text { in probability as } T \rightarrow \infty .
$$

Proof. Choose a real number $\Delta>1 / 2$. Denote $\delta_{\Delta}=T^{-\Delta}$ and $N=\left\lfloor T / \delta_{\Delta}\right\rfloor$, where $\lfloor y\rfloor$ is the usual floor function notation for a real number $y$. Note that $N=O\left(T^{1+\Delta}\right)$ and $N \delta_{\Delta}=O(T)$. Next, let $0=t_{0}<t_{1}<\cdots<t_{N}=T$ be a partition of $[0, T]$ such that $t_{k}=k \delta_{\Delta}$ for $k=0,1, \ldots, N$. For notational simplicity, denote

$$
\begin{aligned}
& \zeta_{i}(t)=\frac{x^{\prime}(t) A_{i} x(t)}{|x(t)|^{2}}, \\
& \widetilde{\zeta}_{i}(t)= \begin{cases}\zeta_{i}(0), & \text { if } 0 \leq t<t_{2}, \\
\zeta_{i}\left(t_{k-1}\right), & \text { if } t_{k} \leq t<t_{k+1}, \quad k=2, \ldots, N-1, \\
\zeta_{i}\left(t_{N-1}\right), & \text { if } t_{N} \leq t \leq T .\end{cases} \\
& I_{i}(t)=I_{\{\gamma(t)=i\}}-\pi_{i} .
\end{aligned}
$$

Note that in the above, $\widetilde{\zeta}_{i}(t)$ is a piecewise constant approximation of $\zeta_{i}(t)$ with the interpolation interval $\left[t_{k}, t_{k+1}\right.$ ) (with the last interval being $\left[t_{N-1}, t_{N}\right]$ ). Using this notation, the assertion of Lemma 4.1 will follow immediately if we can show that for each $i \in \mathcal{M}$,

$$
\mathbf{E}\left|\frac{1}{T} \int_{0}^{T} \zeta_{i}(t) I_{i}(t) d t\right|^{2} \rightarrow 0 \text { as } T \rightarrow \infty .
$$

Using the triangle inequality and the Cauchy-Schwarz inequality, we can readily verify that for each $i \in \mathcal{M}$,

$$
\begin{aligned}
\mathbf{E} \mid & \left.\frac{1}{T} \int_{0}^{T} \zeta_{i}(t) I_{i}(t) d t\right|^{2} \\
& \leq \frac{2}{T^{2}} \mathbf{E}\left|\int_{0}^{T}\left[\zeta_{i}(t)-\widetilde{\zeta}_{i}(t)\right] I_{i}(t) d t\right|^{2}+\frac{2}{T^{2}} \mathbf{E}\left|\int_{0}^{T} \widetilde{\zeta}_{i}(t) I_{i}(t) d t\right|^{2} \\
& \leq \frac{K}{T} \int_{0}^{T} \mathbf{E}\left[\zeta_{i}(t)-\widetilde{\zeta}_{i}(t)\right]^{2} d t+\frac{2}{T^{2}} \mathbf{E}\left|\int_{0}^{T} \widetilde{\zeta}_{i}(t) I_{i}(t) d t\right|^{2} .
\end{aligned}
$$

In the above and hereafter, $K$ is used as a generic positive constant, whose value may change for different appearances. Clearly, for each $i \in \mathcal{M}, \zeta_{i}(\cdot)$ is uniformly bounded by $\left|A_{i}\right|$, the norm of $A_{i}$. Thus

$$
\sup _{0 \leq t \leq T} \mathbf{E}\left|\zeta_{i}(t)\right|^{2}<\infty
$$


Since $x(t) /|x(t)|$ takes values on the unit sphere, it is readily verified that

$$
\sup _{0 \leq t \leq T}\left|\frac{d}{d t} \frac{x^{\prime}(t) A_{i} x(t)}{|x(t)|^{2}}\right| \leq K
$$

where $K$ is independent of $T$. As a result, $\zeta_{i}(\cdot)$ is Lipschitz continuous, uniformly on $[0, T]$. Consequently,

$$
\begin{aligned}
\int_{0}^{T} \mathbf{E}\left[\zeta_{i}(t)-\widetilde{\zeta}_{i}(t)\right]^{2} d t & =\sum_{k=0}^{N-1} \int_{t_{k}}^{t_{k+1}} \mathbf{E}\left[\zeta_{i}(t)-\zeta_{i}\left(t_{k-1}\right)\right]^{2} d t \\
& \leq K \sum_{k=0}^{N-1} \int_{t_{k}}^{t_{k+1}}\left[t-t_{k-1}\right]^{2} d t \\
& =O\left(N \delta_{\Delta}^{3}\right)=O\left(T \delta_{\Delta}^{2}\right) \rightarrow 0 \text { as } T \rightarrow \infty,
\end{aligned}
$$

by the choice of $\delta_{\Delta}$.

By means of (4.4) and (4.6), it remains to show that the last term of (4.4) converges to 0 as $T \rightarrow \infty$. For any $0 \leq t \leq T$, define

$$
h_{i}(t)=\mathbf{E}\left[\int_{0}^{t} \widetilde{\zeta}_{i}(s) I_{i}(s) d s\right]^{2} .
$$

Then

$$
\frac{d h_{i}(t)}{d t}=2 \int_{0}^{t} \mathbf{E}\left[\widetilde{\zeta}_{i}(s) I_{i}(s) \widetilde{\zeta}_{i}(t) I_{i}(t)\right] d s .
$$

It can be seen that for $0 \leq t \leq t_{2}$,

$$
\left|\int_{0}^{t} \mathbf{E}\left[\widetilde{\zeta}_{i}(s) I_{i}(s) \widetilde{\zeta}_{i}(t) I_{i}(t)\right] d s\right| \leq K t_{2}=O\left(T^{-\Delta}\right) .
$$

For $s \leq t_{k-1}<t_{k} \leq t<t_{k+1}$ with $k=2, \ldots, N-2$ and with $k=N-1$ (in this case $\left.t_{N-1} \leq t \leq t_{N}\right)$

$$
\begin{aligned}
\mathbf{E}\left[\widetilde{\zeta}_{i}(s) I_{i}(s) \widetilde{\zeta}_{i}(t) I_{i}(t)\right] & =\mathbf{E}\left[\widetilde{\zeta}_{i}(s) I_{i}(s) \mathbf{E}\left(\widetilde{\zeta}_{i}(t) I_{i}(t) \mid \mathcal{F}_{s}\right)\right] \\
& =\mathbf{E}\left[\widetilde{\zeta}_{i}(s) I_{i}(s) \zeta_{i}\left(t_{k-1}\right) \mathbf{E}\left(I_{i}(t) \mid \mathcal{F}_{s}\right)\right] \\
& =\mathbf{E}\left[\widetilde{\zeta}_{i}(s) I_{i}(s) \zeta_{i}\left(t_{k-1}\right) \mathbf{E}\left(I_{i}(t) \mid \gamma(s)\right)\right] \\
& =\sum_{j=1}^{m} \mathbf{E}\left[\widetilde{\zeta}_{i}(s) I_{i}(s) \zeta_{i}\left(t_{k-1}\right) \mathbf{E}\left(I_{i}(t) \mid \gamma(s)=j\right)\right] .
\end{aligned}
$$

Using [17, Chapter 5], it can be shown that

$$
\left|\mathbf{E}\left[\widetilde{\zeta}_{i}(s) I_{i}(s) \mathbf{E}\left(\widetilde{\zeta}_{i}(t) I_{i}(t) \mid \mathcal{F}_{t_{k-1}}\right)\right]\right|=O\left(\varepsilon+\exp \left(-T^{-\Delta} / \varepsilon\right)\right) .
$$

Using (4.10) in (4.9) and (4.8), we are able to conclude that $\sup _{0 \leq t \leq T}\left|(d / d t) h_{i}(t)\right|<\infty$ for each $i \in \mathcal{M}$. Thus $h_{i}(T) / T^{2} \rightarrow 0$ as $T \rightarrow \infty$. The lemma then follows.

By virtue of Lemma 4.1, we need only consider the last term in (4.3). To this end, for each $i \in \mathcal{M}$, there is a $\lambda_{i} \in \mathbb{R}$ such that

$$
\begin{aligned}
\frac{1}{T} \int_{0}^{T} \frac{x^{\prime}(t) A_{i} x(t)}{|x(t)|^{2}} d t & =\frac{1}{T} \int_{0}^{T} y^{\prime}(t) A_{i} y(t) d t \\
& \rightarrow \lambda_{i} \text { in probability as } T \rightarrow \infty
\end{aligned}
$$


where the existence of the limit follows from a slight modification of [5. p. 344, Theorem 6]. The number $\lambda_{i}$ above is precisely the average of $\zeta_{i}(t)$, known as the mean value of $\zeta_{i}(\cdot)$ and denoted by $M\left[\zeta_{i}\right]$ in the literature of almost periodic functions [5, Appendix]. To find its average, we may use the Fourier series of $\zeta_{i}(t)$ and expand $\zeta_{i}(t)$ as $\zeta_{i}(t)=$ $\sum_{j} z_{i, j} \exp (\mathrm{i}(j t))$, where $z_{i, j}$ 's are the Fourier coefficients corresponding to $\zeta_{i}(t)$ and $\mathrm{i}$ is the imaginary number satisfying $i^{2}=-1$. Define

$$
\lambda=\sum_{i=1}^{m} \pi_{i} \lambda_{i}
$$

Then, it follows from Lemma 4.1 and equations (4.3), (4.11), and (4.12) that

$$
\frac{1}{T} \ln \frac{|x(T)|}{|x(0)|}=\frac{1}{T} \int_{0}^{T} \frac{x^{\prime}(t) A(\gamma(t)) x(t)}{|x(t)|^{2}} d t \rightarrow \lambda \text { as } T \rightarrow \infty .
$$

To proceed, note that (4.13) can be rewritten as

$$
\frac{1}{T} \ln \frac{|x(T)|}{|x(0)|}=\lambda+o(1),
$$

where $o(1) \rightarrow 0$ in probability as $T \rightarrow \infty$. If $\lambda<0$, denote $\lambda=-\lambda_{0}$ with $\lambda_{0}>0$. We have $-\lambda_{0}+o(1) \leq-\lambda_{1}$ where $0<\lambda_{1}<\lambda_{0}$. Thus it follows from (4.14) that

$$
|x(T)| \leq|x(0)| \exp \left(-\lambda_{1} T\right) \rightarrow 0 \text { in probability as } T \rightarrow \infty .
$$

Likewise, if $\lambda>0$, we can find $0<\lambda_{2}<\lambda$ such that $\lambda+o(1) \geq \lambda_{2}$, which in turn, implies that

$$
|x(T)| \geq|x(0)| \exp \left(\lambda_{2} T\right) \rightarrow \infty \text { in probability as } T \rightarrow \infty .
$$

We summarize the result obtained thus far into the following theorem.

Theorem 4.2. The equilibrium point $x=0$ of (4.1) is asymptotically stable in probability if $\lambda<0$ and asymptotically unstable in probability in the sense of (4.15) if $\lambda>0$.

Note that the results in Theorem 4.2 present a dichotomy for the property of the equilibrium point $x=0$ according to $\lambda<0$ or $\lambda>0$. One naturally asks the question: What can we say about the equilibrium point $x=0$ if $\lambda=0$ ? To answer this question, we need the following lemma.

Lemma 4.3. If the equilibrium point $x=0$ of (4.1) is asymptotically stable in probability, then it is exponentially $p$-stable for all sufficiently small positive $p$.

Proof. This lemma can be established using a similar argument as that of $\underline{8}$, Theorem 6.4.1]; we omit the details here for brevity.

With Lemma 4.3 at our hand, we are now able to describe the local qualitative behavior of the equilibrium point $x=0$ when $\lambda=0$.

Theorem 4.4. Suppose that $\lambda=0$. Then the equilibrium point $x=0$ is neither asymptotically stable nor asymptotically unstable in probability.

Proof. Assume first that the equilibrium point $x=0$ is asymptotically stable in probability. Then Lemma 4.3 implies that it is exponentially $p$-stable for all sufficiently small 
positive $p$. Now applying Jensen's inequality to the convex function $\varphi(x)=e^{x}$, from equation (4.3) and Definition 2.1 (iii), we obtain that

$$
\begin{aligned}
& |x(0)|^{p} \exp \left\{p \mathbf{E} \int_{0}^{T} \frac{x^{\prime}(t) A(\gamma(t)) x(t)}{|x(t)|^{2}} d t\right\} \\
& \quad=|x(0)|^{p} \exp \{p \mathbf{E}(\ln |x(T)|-\ln |x(0)|)\} \\
& \quad \leq \mathbf{E}|x(t)|^{p} \leq K|x(0)|^{p} e^{-k T} .
\end{aligned}
$$

Consequently, with positive probability,

$$
\lim _{T \rightarrow \infty} \frac{1}{T} \int_{0}^{T} \frac{x^{\prime}(t) A(\gamma(t)) x(t)}{|x(t)|^{2}} d t<0
$$

According to (4.13), this contradicts the assumption that $\lambda=0$. Thus the equilibrium point $x=0$ is not asymptotically stable. Similarly we can prove that the equilibrium point $x=0$ is not asymptotically unstable in probability if $\lambda=0$.

5. Examples. For simplicity, by a slight abuse of notation, we call a system that is linear with respect to the continuous state a linear system or hybrid linear system. To illustrate, we provide several examples in this section. In addition, we demonstrate results that are associated with the well-known Hartman-Grobman theorem for random switching systems.

EXAmPle 5.1. Consider a system (4.1) (linear in the variable $x$ ) with the following specifications. The Markov chain $\gamma(t)$ has two states and is generated by $Q=$ $\left(\begin{array}{cc}-3 & 3 \\ 1 & -1\end{array}\right)$, and

$$
A_{1}=A(1)=\left(\begin{array}{cc}
-1 & 2 \\
0 & 2
\end{array}\right), \quad A_{2}=A(2)=\left(\begin{array}{cc}
-3 & -1 \\
1 & -2
\end{array}\right) .
$$

Thus associated with the hybrid system (4.1), there are two ordinary differential equations

$$
\begin{aligned}
& \dot{x}(t)=A_{1} x(t) \quad \text { and } \\
& \dot{x}(t)=A_{2} x(t),
\end{aligned}
$$

switching back and forth from one to another according to the movement of the jump process $\gamma(t)$. It is readily seen that the eigenvalues of $A_{1}$ are -1 and 2 . Hence the motion of (5.1) is unstable. Similarly, by computing the eigenvalues of $A_{2}$, the motion of (5.2) is asymptotically stable.

Next we use Corollary 3.3 to show that the hybrid system (4.1) is asymptotically stable in probability owing to the presence of the Markov chain $\gamma(t)$. That is, the Markov chain becomes a stabilizing factor. The stationary distribution of the Markov chain $\gamma(t)$ is $\pi=(0.25,0.75)$, which is obtained by solving the system of equations $\pi Q=0$ and $\pi \mathbb{1}=1$. The maximum eigenvalues of $A_{1}+A_{1}^{\prime}$ and $A_{2}+A_{2}^{\prime}$ are

$$
\lambda_{\max }\left(A_{1}+A_{1}^{\prime}\right)=4.6056, \quad \lambda_{\max }\left(A_{2}+A_{2}^{\prime}\right)=-4,
$$

respectively. This yields that

$$
\pi_{1} \lambda_{\max }\left(A_{1}+A_{1}^{\prime}\right)+\pi_{2} \lambda_{\max }\left(A_{2}+A_{2}^{\prime}\right)=-1.8486<0 .
$$


Therefore, we conclude from Corollary 3.3 that the hybrid system (4.1) is asymptotically stable in probability. The phase portrait Figure 1(a) confirms our findings. It is interesting to note the dynamic movements and the interactions of continuous components and the discrete component. To see the difference between hybrid system and ordinary differential equations, we also present the phase portraits of (5.1) and (5.2) in Figure1(b).

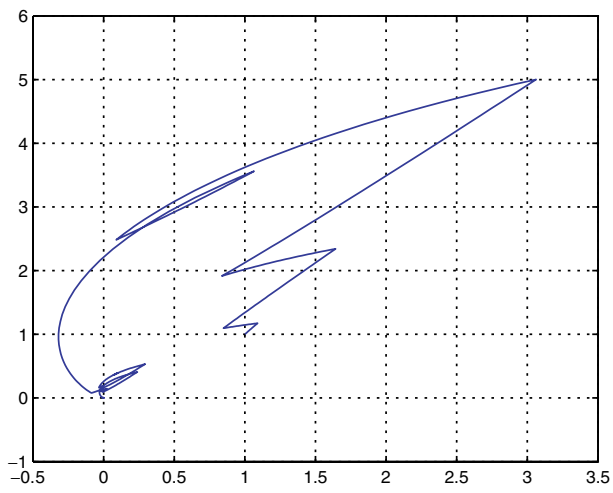

(a) Hybrid linear system: Phase portrait of (4.1) with the initial condition $(x, \gamma)=$ $\left([1,1]^{\prime}, 1\right)$

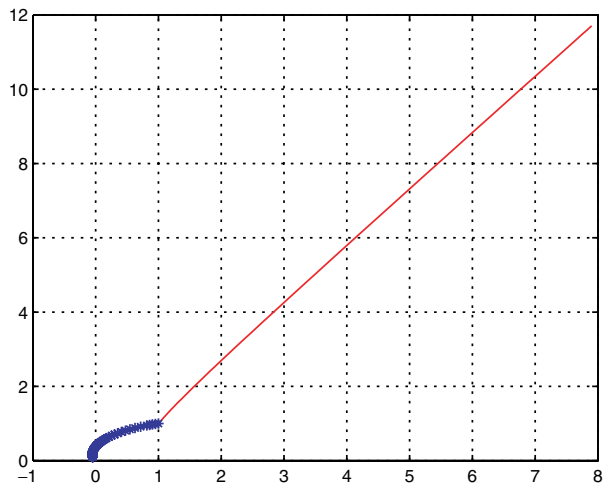

(b) Phase portraits of (5.1) (in the solid line) and (5.2) (in the starred line) with the same initial condition $x=[1,1]^{\prime}$

FIG. 1. Comparisons of the switching system and the associated ordinary differential equations

EXAmple 5.2. Consider a system (linear in the $x$ variable)

$$
\dot{x}(t)=A(\gamma(t)) x(t)
$$

with the following specifications. Here we consider an $x$-dependent generator $Q(x)$. The discrete event process $\gamma(t)$ has two states and is generated by

$$
Q(x)=Q\left(x_{1}, x_{2}\right)=\left(\begin{array}{cc}
-2-\sin x_{1} \cos \left(x_{1}^{2}\right)-\sin \left(x_{2}^{2}\right) & 2+\sin x_{1} \cos \left(x_{1}^{2}\right)+\sin \left(x_{2}^{2}\right) \\
1-0.5 \sin \left(x_{1} x_{2}\right) & -1+0.5 \sin \left(x_{1} x_{2}\right)
\end{array}\right)
$$

and

$$
A_{1}=A(1)=\left(\begin{array}{ll}
0 & 0 \\
3 & 0
\end{array}\right), \quad A_{2}=A(2)=\left(\begin{array}{cc}
-1 & 2 \\
-2 & -1
\end{array}\right) .
$$

Note that the distinct feature of this example compared with the last one is that the $Q$ matrix is $x$ dependent, which satisfies the approximation condition posed in Section 4 . Associated with the hybrid system (4.1), there are two ordinary differential equations

$$
\begin{aligned}
& \dot{x}(t)=A_{1} x(t) \quad \text { and } \\
& \dot{x}(t)=A_{2} x(t),
\end{aligned}
$$

switching from one to another according to the movement of the jump process $\gamma(t)$. Solving (5.4), we obtain

$$
x_{1}(t)=c_{1}, \quad x_{2}(t)=3 c_{1} t+c_{2}
$$


for some constants $c_{1}$ and $c_{2}$. Hence the motion of (5.4) is unstable if the initial point $\left(c_{1}, c_{2}\right)$ is not in the $y$-axis. Meanwhile, by computing the eigenvalues of $A_{2}$, we obtain that the motion of (5.5) is asymptotically stable.

By virtue of Corollary 3.3 , the hybrid system (5.3) is asymptotically stable in probability due to the stabilizing jump process $\gamma(t)$. We first note that as $x \rightarrow 0$,

$$
Q(x) \rightarrow \widehat{Q}=\left(\begin{array}{cc}
-2 & 2 \\
1 & -1
\end{array}\right),
$$

and hence the stationary distribution of the Markov chain $\widehat{\gamma}(t)$ is $\pi=(1 / 3,2 / 3)$. The maximum eigenvalues of $A_{1}+A_{1}^{\prime}$ and $A_{2}+A_{2}^{\prime}$ are

$$
\lambda_{\max }\left(A_{1}+A_{1}^{\prime}\right)=3, \quad \lambda_{\max }\left(A_{2}+A_{2}^{\prime}\right)=-2,
$$

respectively. It follows that

$$
\pi_{1} \lambda_{\max }\left(A_{1}+A_{1}^{\prime}\right)+\pi_{2} \lambda_{\max }\left(A_{2}+A_{2}^{\prime}\right)=-1 / 3<0 .
$$

Therefore, we conclude from Corollary 3.3 that the hybrid system (5.3) is asymptotically stable in probability. The phase portrait Figure 2 (a) confirms our results. To delineate the difference between the hybrid system and ordinary differential equations, we also present the phase portraits of (5.4) and (5.5) in Figure 2(b). The phase portraits reveal the interface of continuous and discrete components. They illustrate the hybrid characteristics in an illuminating way.

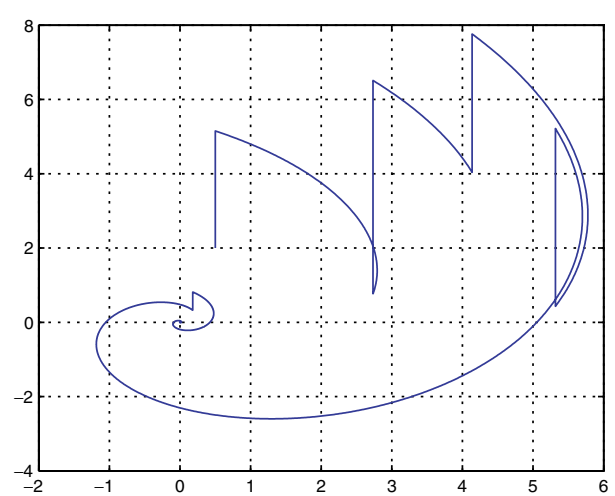

(a) Hybrid linear system: Phase portrait of (5.3) with the initial condition $(x, \gamma)=$ $\left([0.5,2]^{\prime}, 1\right)$

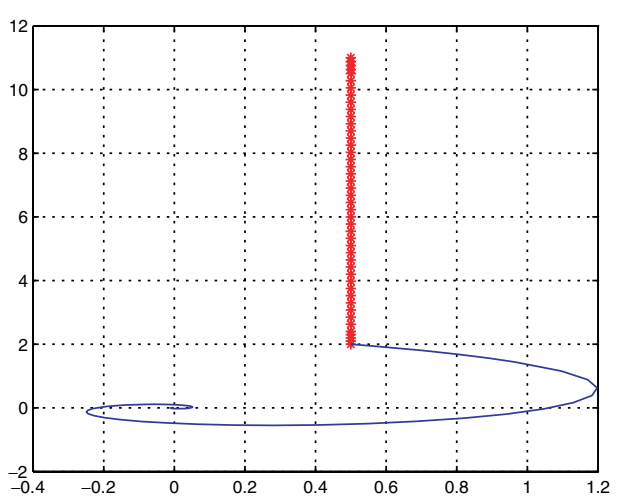

(b) Phase portraits of (5.4) (in the starred line) and (5.5) (in the solid line) with the same initial condition $x=[0.5,2]^{\prime}$

FIG. 2. Comparisons of the switching system and the associated ordinary differential equations

EXAMPLE 5.3. In this example, we consider a nonlinear hybrid system

$$
\dot{x}(t)=f(x(t), \gamma(t)),
$$


where $x(t)$ is a two-dimensional state trajectory, $\gamma(t)$ is a jump process taking value in $\mathcal{M}=\{1,2,3\}$ with a generator $Q(x)$ given by

$$
\left(\begin{array}{ccc}
\sin x_{2}-2-\cos x_{1} & 1-\sin x_{2} & 1+\cos x_{1} \\
1-0.5 \sin \left(x_{1} x_{2}\right) & 0.5 \sin \left(x_{1} x_{2}\right)-1-\cos ^{2}\left(x_{1} x_{2}\right) & \cos ^{2}\left(x_{1} x_{2}\right) \\
0 & 3+\sin x_{1}+\sin x_{2} \cos x_{2} & -3-\sin x_{1}-\sin x_{2} \cos x_{2}
\end{array}\right) .
$$

The functions $f(x, i), i=1,2,3$, are defined as:

$$
\begin{aligned}
& f(x, 1)=\left[\begin{array}{c}
x_{1}+\frac{x_{1}^{2}}{1+x_{1}^{2}+x_{2}^{2}} \\
-2 x_{2}+\sin \left(x_{1} x_{2}\right) \cos x_{2}
\end{array}\right], \\
& f(x, 2)=\left[\begin{array}{c}
-2 x_{1}+x_{2}-2 x_{1} \sin x_{2} \\
-x_{1}-x_{2}+x_{1} \cos x_{1} \sin x_{2}
\end{array}\right], \\
& f(x, 3)=\left[\begin{array}{c}
x_{2}+2 x_{1} \cos x_{1} \sin x_{2} \\
-x_{1}+x_{2} \sin x_{1}
\end{array}\right] .
\end{aligned}
$$

Note that the matrices $\widehat{Q}$, and $A_{i}, i \in \mathcal{M}$, in assumption (A4) can be obtained as follows

$$
\widehat{Q}=\left(\begin{array}{ccc}
-3 & 1 & 2 \\
1 & -1 & 0 \\
0 & 3 & -3
\end{array}\right)
$$

and

$$
A_{1}=\left(\begin{array}{cc}
1 & 0 \\
0 & -2
\end{array}\right), \quad A_{2}=\left(\begin{array}{cc}
-2 & 1 \\
-1 & -1
\end{array}\right), \text { and } A_{3}=\left(\begin{array}{cc}
0 & 1 \\
-1 & 0
\end{array}\right)
$$

For a system of differential equations without switching, the well-known HartmanGrobman theorem holds. It indicates that for hyperbolic equilibria, a linear system arising from approximation is topologically equivalent to the associated nonlinear systems, whereas a system with a center is not. When random switching appears in the system, we show that the nonlinear system and its approximation could be equivalent even if equilibria with center in one or more of its components as long as the component with hyperbolic equilibrium dominates the rest of them.

We proceed to use Theorem 3.2 and Corollary 3.3 to verify that the hybrid system (5.6) is asymptotically stable in probability. The stationary distribution of the Markov chain $\widehat{\gamma}(t)$ is $\pi=(3 / 14,9 / 14,1 / 7)$. Now consider the symmetric and positive definite matrix $G=\left(\begin{array}{cc}3 & -1 \\ -1 & 3\end{array}\right)$ and compute the maximum eigenvalues of the matrices $G A_{i} G^{-1}+$ $G^{-1} A_{i}^{\prime} G$ with $i=1,2,3$,

$$
\lambda_{\max }\left(G A_{1} G^{-1}+G^{-1} A_{1}^{\prime} G\right)=\frac{11}{4}, \quad \lambda_{\max }\left(G A_{2} G^{-1}+G^{-1} A_{2}^{\prime} G\right)=-\frac{11}{4}
$$

and

$$
\lambda_{\max }\left(G A_{3} G^{-1}+G^{-1} A_{3}^{\prime} G\right)=\frac{3}{2} .
$$

Thus we obtain

$$
\sum_{i=1}^{3} \pi_{i} \lambda_{\max }\left(G A_{i} G^{-1}+G^{-1} A_{i}^{\prime} G\right)=-\frac{27}{28}<0
$$


Hence Theorem 3.2 implies that the hybrid system (5.6) is asymptotically stable in probability.

It is interesting to note that the linear approximation

$$
\dot{x}(t)=A(\widehat{\gamma}(t)) x(t)
$$

is also asymptotically stable in probability, where the matrices $A(i)=A_{i}, i \in \mathcal{M}$, are as in (5.8), and the Markov chain $\widehat{\gamma}(t)$ is generated by $\widehat{Q}$ in (5.7). To demonstrate, we present the phase portrait of (5.6) in Figure 3(a), whereas Figure 3(b) presents the phase portrait of its first order linear approximation (5.9).

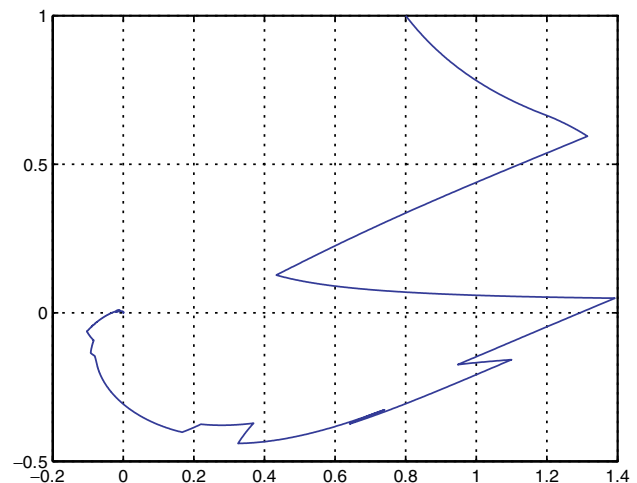

(a) Hybrid nonlinear system: Phase portrait of (5.6) with the initial condition $(x, \gamma)=\left([0.8,1]^{\prime}, 1\right)$

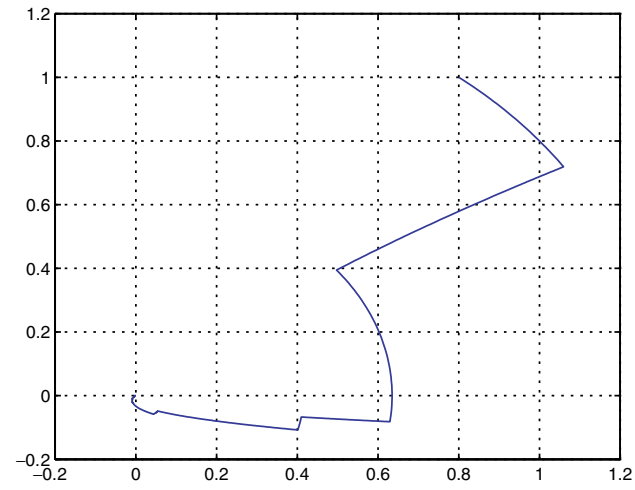

(b) Hybrid linear system: Phase portrait of $[5.9$ with the initial condition $(x, \gamma)=\left([0.8,1]^{\prime}, 1\right)$

FiG. 3. Comparisons of the nonlinear system and its linear approximation

6. Further remarks. This work has been focused on the stability and instability of random switching systems of differential equations. Sufficient conditions are derived. These conditions are easily verifiable and are based on coefficients of the systems. For systems that are linear in the continuous component, certain necessary and sufficient conditions are derived via a transformation technique, which closes the gap in using maximal and minimal eigenvalues of certain matrices. Somewhat remarkably, a particular interesting discovery is: Different from a single system of differential equations, in which the Hartman-Grobman theorem is in force, for switching systems of differential equations modulated by a random process, even if some of the equilibria are not hyperbolic (e.g., center), the original system and that of the "linearized" (with respect to the continuous variable) system still have the same asymptotic behavior. This is demonstrated by our analytical results as well as computations with the use of phase portraits.

\section{REFERENCES}

[1] G. Barone-Adesi and R. Whaley, Efficient analytic approximation of American option values, Journal of Finance, 42 (1987), 301-320. 
[2] G.K. Basak, A. Bisi, and M.K. Ghosh, Stability of a random diffusion with linear drift, J. Math. Anal. Appl., 202 (1996), 604-622. MR.1406250 (97g:60091)

[3] P. Billingsley, Convergence of Probability Measures, J. Wiley \& Sons, Inc., New York, 1968. MR0233396 (38:1718)

[4] M.H.A. Davis, Piecewise-deterministic Markov processes: a general class of nondiffusion stochastic models, J. Roy. Statist. Soc. Ser. B, 46(3) (1984), 353-388. MR.790622 (87g:60062)

[5] J. Hale, Ordinary Differential Equations, 2nd Ed., R.E. Krieger Pub. Co., Malabar, FL, 1980. MR $587488(82 \mathrm{e}: 34001)$

[6] I.Ia. Kac and N.N. Krasovskii, On the stability of systems with random parameters, J. Appl. Math. Mech., 24 (1960), 1225-1246.

[7] Y. Ji and H.J. Chizeck, Controllability, stabilizability, and continuous-time Markovian jump linear quadratic control, IEEE Trans. Automatic Control, 35 (1990), 777-788. MR1058362 (91h:49037)

[8] R.Z. Khasminskii, Stochastic Stability of Differential Equations, Sijthoff and Noordhoff, Alphen aan den Rijn, Netherlands, 1980. MR600653 (82b:60064)

[9] R.Z. Khasminskii, C. Zhu, and G. Yin, Stability of regime-switching diffusions, Stochastic Process. Appl., 117 (2007), 177-194.

[10] H.J. Kushner and G. Yin, Stochastic Approximation and Recursive Algorithms and Applications, 2nd Ed., Springer-Verlag, New York, NY, 2003. MR.1993642 (2004e:62005)

[11] X. Mao, Stability of stochastic differential equations with Markovian switching, Stochastic Process. Appl., 79 (1999), 45-67. MR1666831 (2000e:60095)

[12] M. Mariton, Jump Linear Systems in Automatic Control, Marcel Dekker, Inc., New York, 1990.

[13] L. Perko, Differential Equations and Dynamical Systems, Springer, 3rd Ed., New York, 2001. MR 1801796 (2001k:34001)

[14] A.V. Skorohod, Asymptotic Methods in the Theory of Stochastic Differential Equations, Amer. Math. Soc., Providence, RI, 1989. MR1020057 (90i:60038)

[15] G. Yin, and V. Krishnamurthy, Least mean square algorithms with Markov regime switching limit, IEEE Trans. Automat. Control, 50 (2005), 577-593. MR2141561 (2006b:93245)

[16] G. Yin, V. Krishnamurthy, and C. Ion, Regime switching stochastic approximation algorithms with application to adaptive discrete stochastic optimization, SIAM J. Optim., 14 (2004), 1187-1215. MR2112970 (2005m:62148)

[17] G. Yin and Q. Zhang, Continuous-Time Markov Chains and Applications: A Singular Perturbation Approach, Springer-Verlag, New York, 1998. MR1488963(2000a:60142)

[18] G. Yin and C. Zhu, On the notion of weak stability and related issues of hybrid diffusion systems, Nonlinear Anal.: Hybrid Sys., 1 (2007), 173-187.

[19] X.Y. Zhou and G. Yin, Markowitz's mean-variance portfolio selection with regime switching: $A$ continuous-time model, SIAM J. Control Optim., 42 (2003), 1466-1482. MR2044805 (2004m:91130)

[20] C. Yuan and X. Mao, Asymptotic stability in distribution of stochastic differential equations with Markovian switching, Stochastic Process Appl., 103 (2003), 277-291. MR1950767 (2003k:60142) 